\title{
Intrinsic development of choroidal and thalamic collaterals in hemorrhagic-onset moyamoya disease: case-control study of the Japan Adult Moyamoya Trial
}

\author{
Miki Fujimura, MD, PhD, ${ }^{1}$ Takeshi Funaki, MD, PhD, ${ }^{2}$ Kiyohiro Houkin, MD, PhD, ${ }^{3}$ \\ Jun C. Takahashi, MD, PhD, ${ }^{4}$ Satoshi Kuroda, MD, PhD, ${ }^{5}$ Yasutake Tomata, $\mathrm{PhD},{ }^{6}$ \\ Teiji Tominaga, MD, PhD, ${ }^{1}$ Susumu Miyamoto, MD, $\mathrm{PhD},{ }^{2}$ on behalf of the Japan Adult Moyamoya \\ Trial Investigators
}

\begin{abstract}
'Department of Neurosurgery, Tohoku University Graduate School of Medicine, Sendai; 'Department of Neurosurgery, Kyoto University Graduate School of Medicine, Kyoto; ${ }^{3}$ Department of Neurosurgery, Hokkaido University Graduate School of Medicine, Sapporo; ${ }^{4}$ Department of Neurosurgery, National Cerebral and Cardiovascular Center, Suita; ${ }^{5}$ Department of Neurosurgery, Graduate School of Medicine and Pharmaceutical Sciences, University of Toyama, Toyama; and ${ }^{6}$ Division of Epidemiology, Department of Health Informatics and Public Health, Tohoku University School of Public Health, Graduate School of Medicine, Sendai, Japan
\end{abstract}

OBJECTIVE This study was performed to identify the angiographic features of hemorrhagic-onset moyamoya disease (MMD) in comparison with those of patients with ischemic-onset MMD.

METHODS This case-control study compared the data set of the Japan Adult Moyamoya (JAM) Trial with the angiographic data of adult patients with ischemic-onset MMD. The authors analyzed angiograms obtained at onset, classifying the collaterals into 3 subtypes: lenticulostriate anastomosis, thalamic anastomosis, and choroidal anastomosis. They then compared the extent of these collaterals, as indicated by the collateral development grade from 0 to 2 in each subtype, between the JAM Trial group and the ischemic-onset group. They also compared the involvement of the posterior cerebral artery (PCA) and Suzuki's angiographic staging between each group.

RESULTS Among 89 ischemic-onset patients, 103 symptomatic hemispheres in 80 patients were analyzed and compared with 75 hemorrhagic hemispheres from the JAM Trial. The hemorrhagic-onset patients showed a significantly higher proportion of thalamic anastomosis $(p=0.043)$ and choroidal anastomosis $(<0.001)$, as indicated by grade 2 in each subtype, compared with ischemic-onset patients. Suzuki's angiographic staging was significantly higher in the hemorrhagic group (<0.038). There was no difference in the extent of lenticulostriate anastomosis and PCA involvement between the groups.

CONCLUSIONS In adult MMD, the characteristic pattern of the abnormal vascular networks at the base of the brain is different between each onset type. In light of the more prominent development of thalamic and choroidal anastomosis in the JAM Trial group in the present study, development of these collaterals, especially the choroidal collateral extending beyond the lateral ventricle, may play a critical role in hemorrhagic presentation in MMD.

Clinical trial registration no. C000000166 (http://www.umin.ac.jp/ctr/index.htm)

https://thejns.org/doi/abs/10.3171/2017.11.JNS171990

KEYWORDS EC-IC; extracranial-intracranial; moyamoya disease; intracerebral hemorrhage; cerebral ischemia; cerebral angiography; vascular disorders

$\mathrm{M}$ OYAMOYA disease (MMD) is a chronic cerebrovascular disease with unknown etiology, characterized by bilateral stenoocclusive changes at the terminal portion of the internal carotid artery (ICA) and an abnormal vascular network development at the base of the brain. ${ }^{5,15,16}$ MMD has a unique and dynamic compensatory nature to complement its ischemic condition, as indicated by Suzuki's angiographic staging. ${ }^{4,16}$ Insufficiency of this compensatory reorganization system may result not only in cerebral ischemia, but also in intracranial hemorrhage

ABBREVIATIONS EC-IC = extracranial-intracranial; ICA = internal carotid artery; JAM = Japan Adult Moyamoya; $M M D=$ moyamoya disease; $P C A=$ posterior cerebral artery.

SUBMITTED August 12, 2017. ACCEPTED November 28, 2017.

INCLUDE WHEN CITING Published online May 4, 2018; DOI: 10.3171/2017.11.JNS171990. 
due to inadequate collateral vascular networks. ${ }^{3}$ However, the mechanism by which the intracranial hemorrhage occurs at abnormal collaterals is still undetermined.

The Japan Adult Moyamoya (JAM) Trial was a unique randomized controlled trial that demonstrated the efficacy of direct extracranial-intracranial (EC-IC) bypass for hemorrhagic-onset MMD. ${ }^{13}$ Prespecified subgroup analysis of the JAM Trial data further indicated that patients with posterior circulation hemorrhage had a much higher rebleeding rate (17.1\%) compared with that in those with anterior circulation hemorrhage, and EC-IC bypass significantly reduced the risk of rebleeding in patients with posterior circulation hemorrhage. ${ }^{17}$ Furthermore, the most recent cross-sectional study using the data set of the JAM Trial suggested that two features, the development of choroidal anastomosis and posterior cerebral artery (PCA) involvement, are characteristic of posterior circulation hemorrhage in MMD? ${ }^{7}$ Therefore, the collateral pattern of MMD could be a critical factor for determining the onset type, either ischemic or hemorrhagic, but the exact difference of these collateral developments between ischemiconset and hemorrhagic-onset patients is still unclear.

The objective of this case-control study was to clarify the characteristic angioarchitecture of hemorrhagic-onset MMD. We sought to compare the data set of the JAM Trial with the angiographic data of adult patients with ischemiconset MMD obtained from compatible institutes.

\section{Methods}

The study was approved by the ethical committees of all participating centers and was registered with the University Hospital Medical Information Network Clinical Trials Registry (no. C000000166).

\section{Participants and Setting}

Angiographic data of hemorrhagic-onset MMD patients were derived from the data set available at the time of enrollment in the JAM Trial. Detailed information on the participants and setting of the JAM Trial has been presented elsewhere. ${ }^{13}$ Participants were recruited from 22 centers: 14 academic, 5 national or public, and 3 private. Patients with MMD were eligible for the study if they had experienced intracranial hemorrhage within the 12 months before randomization, were aged 16 to 65 years, were independent in daily life (score of 0 to 2 on the modified Rankin Scale), had completed acute-phase treatment at least 1 month before randomization, and had been free from ischemic/hemorrhagic attack for at least 1 month. Patients with ruptured circle of Willis aneurysms were excluded. MMD was diagnosed according to the guideline proposed in 1997. ${ }^{5}$

Angiographic data obtained from ischemic-onset MMD patients, presenting with either transient ischemic attack or minor completed stroke, were recruited from 4 academic centers, all of which had participated in the JAM Trial. Patients were aged 16 to 65 years and were independent in daily life (score of 0 to 2 on the modified Rankin Scale), and were therefore compatible with the inclusion criteria of the JAM Trial. We then collected angiographic data of 89 patients who had undergone catheter angiography during the compatible period of the JAM Trial and were diagnosed as having MMD before the completion of the JAM Trial.

\section{Classification and Measurement of Abnormal Collaterals}

The primary factor of interest in the present analysis was to identify the differences of the abnormal collateral vessels in the basal and periventricular region between ischemic-onset and hemorrhagic-onset MMD patients. $\mathrm{Pa}-$ tients were evaluated by catheter angiography at the onset of hemorrhage or ischemic symptoms. The present study employed an angiographic classification system and grading for basal and periventricular collaterals, as introduced in our previous study (Table 1). ${ }^{7}$ Briefly, collateral vessels were classified into 3 types: lenticulostriate anastomosis, thalamic anastomosis, and choroidal anastomosis. The image-determination committee, which included 5 members (M.F., T.F., J.C.T., S.K., and S.M.), recorded the presence or absence of each type of collateral in each hemisphere according to the criteria outlined below. All 5 members of the image-determination committee attended the discussion on the angiographic findings in all cases, and the angiographic grades in each collateral type were ultimately decided after we achieved unanimous agreement of the particular grade.

\section{Lenticulostriate Anastomosis}

This type is anatomically defined as an anastomosis between the lenticulostriate artery and the medial end of the medullary artery, serving as a supply route to the cortex. ${ }^{7}$ We defined grade 0 as no dilation and no extension of the lenticulostriate arteries, grade 1 as dilation and extension of the lenticulostriate arteries below the level of the pericallosal artery, and grade 2 as dilation and extension of lenticulostriate arteries beyond the level of the pericallosal artery in the lateral view. Grade 2 was ultimately considered to be a positive angiographic indicator of lenticulostriate anastomosis.

\section{Thalamic Anastomosis}

This type of collateral is defined as an anastomosis between the thalamic perforators and the medial end of the medullary artery or the insular artery, serving as a supply route to the cortex. The thalamotuberal artery, arising from the posterior communicating artery, and the thalamoperforating artery, arising from the PCA, are the origins of such an anastomosis. We defined grade 0 as no dilation and no extension of the thalamic perforators, grade 1 as dilation and extension of the thalamic perforators below the level of the medial posterior choroidal artery, and grade 2 as dilation and extension of thalamic perforators beyond the level of the medial posterior choroidal artery. Grade 2 was ultimately considered to be a positive angiographic indicator of thalamic anastomosis.

\section{Choroidal Anastomosis}

This type of collateral is typically defined as an anastomosis between the choroidal artery and the medial end of the medullary artery. ${ }^{7}$ Both the anterior and lateral posterior choroidal arteries can serve as the origin of such an anastomosis. We defined grade 0 as no dilation and no extension of the choroidal artery, grade 1 as dilation and 
TABLE 1. Angiographic scoring of lenticulostriate, thalamic, and choroidal anastomosis in MMD

\begin{tabular}{llll}
\hline \multicolumn{1}{c}{ Collateral } & \multicolumn{1}{c}{ Grade 0} & Grade 1 & Grade 2 \\
\hline Lenticulostriate & No dilation, no extension & $\begin{array}{l}\text { Dilation/extension below the level of the perical- } \\
\text { losal artery }\end{array}$ & $\begin{array}{c}\text { Dilation/extension beyond the level of the perical- } \\
\text { losal artery }\end{array}$ \\
\hline Thalamic (perforator) & No dilation, no extension & $\begin{array}{l}\text { Dilation/extension below the level of the MPChA } \\
\text { Dilation/extension beyond the level of the MPChA }\end{array}$ \\
\hline Choroidal & No dilation, no extension & $\begin{array}{l}\text { Dilation/extension below the level of the lateral } \\
\text { ventricle }\end{array}$ & $\begin{array}{l}\text { Dilation/extension beyond the level of lateral } \\
\text { ventricle }\end{array}$ \\
\hline
\end{tabular}

MPChA = medial posterior choroidal artery

extension of the choroidal artery below the level of the lateral ventricle, and grade 2 as dilation and extension of the choroidal artery beyond the level of the lateral ventricle. Grade 2 was ultimately considered to be a positive angiographic indicator of choroidal anastomosis. A positive angiographic (lateral view) indicator of grade 2 is extreme dilation and extension of the choroidal artery with sudden deviation from the level of the lateral ventricle at its peripheral portion to connect to the medullary artery? In the anteroposterior view, this collateral is similar to that in lenticulostriate anastomosis in that it has a typical sharp

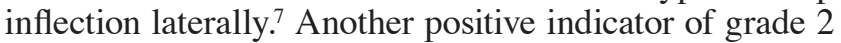
is the extreme extension of the anterior choroidal or lateral posterior choroidal artery beyond the atrium of the lateral ventricle to reach the body of the lateral ventricle. Regarding the medial posterior choroidal artery, grade 2 is considered positive when the artery connects to the pericallosal artery by penetrating the corpus callosum.

Angiographic findings in representative cases of each onset group are shown in Fig. 1. A 49-year-old man presenting with hemorrhage showed grade 2 of both choroidal (asterisks in Fig. 1A and B) and thalamic anastomosis (arrows in Fig. 1A and B), while lenticulostriate anastomosis was grade 1. A 28-year-old woman presenting with a transient ischemic attack had grade 2 lenticulostriate anastomosis (arrow in Fig. 1C and D), while her grades of thalamic and choridal anastomosis were 0 and 1 , respectively.

\section{Other Variables}

The patients' age and sex were collected as baseline data. The image-determination committee also evaluated the involvement of the PCA, a candidate for a potential confounder, and Suzuki's angiographic staging, and they compared data between each group. PCA involvement was categorized into grade 0,1 , and 2 . We defined grade 0 as no stenoocclusive change in the PCA; grade 1 as the presence of occlusion or stenosis greater than $50 \%$ in the $\mathrm{P}_{1}-\mathrm{P}_{3}$ segments of the PCA, with decreased delineation of the cortical arteries; and grade 2 as occlusion of the PCA. The PCA involvement grade and Suzuki's angiographic stage were recorded for each hemisphere. ${ }^{17}$

\section{Statistical Analysis}

Information pertaining to the hemorrhagic hemisphere (the hemisphere in which the hemorrhage had supposedly occurred in each patient) and the hemisphere with ischemic symptoms was extracted for the statistical analysis.

Patients for whom the hemorrhagic hemisphere could not be determined were excluded from the analysis. If the angioarchitecture of the hemisphere with ischemic symptoms was atypical-for example, stenoocclusive change was absent at the terminal ICA-the case was excluded from the analysis. The proportion of angiographic indicator (grade 2) of lenticulostriate anastomosis, thalamic anastomosis, and choroidal anastomosis was compared between ischemic-onset and hemorrhagic-onset groups by the chi-square test. The PCA involvement score and Suzuki's angiographic stage were also compared between each onset group (chi-square test). As for comparison of the baseline characteristics, t-tests and chi-square tests were used as appropriate. Multiple logistic regression models were used to adjust for age and sex. Two-sided values of $\mathrm{p}$ $<0.05$ or $95 \%$ CIs not including 1 were considered to be significant. All analyses were performed with IBM SPSS (version 24, IBM Corp.).

\section{Results}

Among the total of 80 hemorrhagic-onset patients enrolled in the JAM Trial, 4 were excluded from the present analysis because the hemisphere in which their hemorrhage occurred could not be determined; specifically, in 3 of these patients the hemorrhage occurred on the midline, and in 1 patient the diffusely distributed intraventricular hemorrhage occurred in the bilateral ventricles. One additional patient was excluded because the original angiography studies of that patient had been lost. Accordingly, 75 hemorrhagic hemispheres in 75 patients were included in the actual analysis. Regarding 89 ischemic-onset patients recruited to this case-control study, 9 were excluded because of atypical angiographic findings in the symptomatic hemisphere, such as the absence of ICA terminal stenosis and/or the absence of abnormal collateral development. Twenty-three patients presented with ischemic symptoms bilaterally. Accordingly, 103 ischemic hemispheres of 80 patients were included in the actual analysis.

\section{Patients' Background}

As shown in Table 2, the percentage of females was $69.9 \%$ in the ischemic-onset and $68.0 \%$ in the hemorrhagic-onset group, and there was no statistical difference between the groups $(\mathrm{p}=0.786)$. The patients' age $( \pm \mathrm{SD})$ was significantly lower in the ischemic-onset group (35.8 \pm 11.2 years) than in the hemorrhagic-onset group (41.5 \pm 11.4 years $)(\mathrm{p}<0.001)$.

\section{Angiographic Score of Each Collateral Pattern}

The proportion of high angiographic scores, as indicated by grade 2 , was compared between ischemic-onset and 


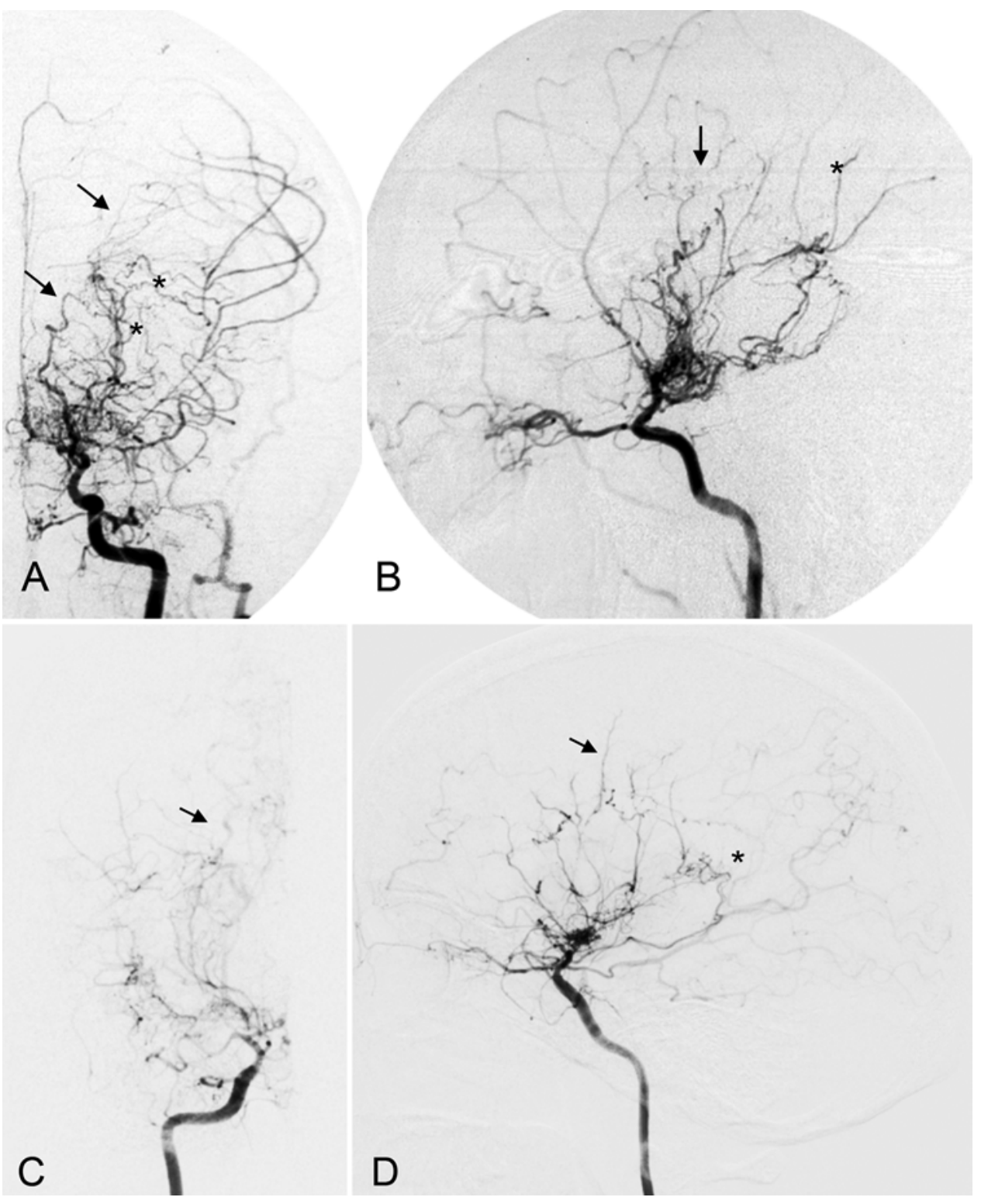

FIG. 1. Angiographic findings of representative cases of each onset type. A and B: A 49-year-old man with hemorrhage. A carotid artery angiogram indicated a dilated choroidal artery extending beyond the lateral ventricle (asterisks in A and B). The thalamic perforators are also dilated and extended (arrows in A and B). C and D: A 28-year-old woman presenting with a transient ischemic attack. A carotid artery angiogram demonstrated lenticulostriate arteries extending beyond the level of the pericallosal artery (arrows in $\mathrm{C}$ and D). The choroidal artery was dilated, but its extension was below the level of the lateral ventricle (asterisk in D).

hemorrhagic-onset patients (Fig. 2). Grade 2 lenticulostriate anastomosis was seen in $22.3 \%$ of the ischemic-onset patients and in $28.0 \%$ of the hemorrhagic-onset patients, and there was no statistically significant difference between the groups $(\mathrm{p}=0.387)$. The incidence of grade 2 thalamic anastomosis was significantly higher in the hem-

TABLE 2. Summary of demographics

\begin{tabular}{lccr}
\hline \multirow{2}{*}{ Characteristic } & \multicolumn{2}{c}{ Presentation } & \multirow{2}{p}{} \\
\cline { 2 - 3 } Value \\
\hline Female, $\%$ & $69.9 \%$ & $68.0 \%$ & 0.786 \\
\hline Age (yrs), mean \pm SD & $35.8 \pm 11.2$ & $41.5 \pm 11.4$ & $<0.001$ \\
\hline
\end{tabular}

orrhagic-onset group (29.3\%) than in the ischemic-onset group $(16.5 \%)(\mathrm{p}=0.043)$. The incidence of grade 2 choroidal anastomosis was significantly higher in the hemorrhagic-onset group (46.7\%) than in the ischemic-onset group $(16.5 \%)(\mathrm{p}<0.001)$. After the age/sex adjustments, odds ratios of grade 2 thalamic anastomosis $(\mathrm{p}=0.041)$ and grade 2 choroidal anastomosis $(\mathrm{p}<0.001)$ remained significantly higher in the hemorrhagic-onset group than in the ischemic-onset group (Table 3).

\section{Involvement of the PCA and Suzuki's Angiographic Staging}

The PCA involvement score was compared between ischemic-onset and hemorrhagic-onset groups, and no 


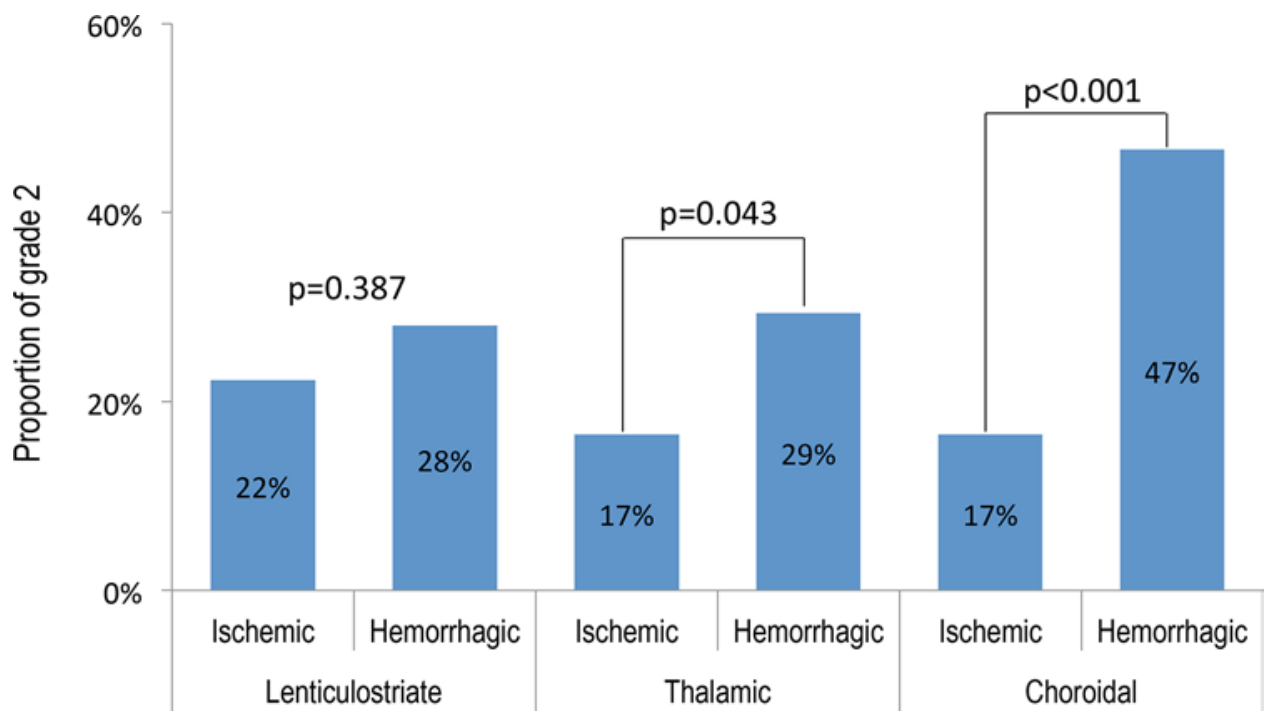

FIG. 2. The prevalence of high angiographic score (grade 2) according to each collateral pattern. Statistical significance was determined by chi-square test. Figure is available in color online only.

significant difference was found (Table 4). As shown in Table 5, the proportion of patients with advanced Suzuki's angiographic staging (stage 4-6) was significantly higher in the hemorrhagic-onset group $(68.0 \%)$ than that in the ischemic-onset group $(52.4 \%)(\mathrm{p}<0.038)$. After the age/ sex adjustments, odds ratios of advanced Suzuki's angiographic staging (stage 4-6) remained higher in the hemorrhagic-onset group $(\mathrm{p}=0.045)$ than those in the ischemiconset group (Table 5).

\section{Discussion}

The present study demonstrated in adult MMD that hemorrhagic-onset patients showed a significantly higher incidence of thalamic anastomosis $(p=0.043)$ and choroidal anastomosis $(<0.001)$ compared with ischemic-onset patients, suggesting that the characteristic pattern of abnormal vascular networks at the base of the brain is apparently distinct between each onset type of adult MMD. The result also showed that Suzuki's angiographic stage was significantly higher in the hemorrhagic-onset group than that in the ischemic-onset group $(\mathrm{p}<0.038)$. In light of more prominent developments of thalamic and choroidal anastomosis in the JAM Trial group in the present study, development of these collaterals, especially the choroidal collateral extending beyond the lateral ventricle, may play a critical role in hemorrhagic presentation in MMD.

The results of our present study are in accordance with those of previous studies, underscoring the significance of the choroidal arteries in hemorrhagic-onset MMD., ${ }^{7,10}$ The high prevalence of choroidal artery dilation in hemorrhagic-onset MMD patients was first reported by Irikura and colleagues in their comparative angiographic study of 10 hemorrhagic-onset and 9 ischemic-onset adult patients with MMD.$^{10}$ Morioka et al. further compared their angiographic findings in a larger series including 37 ischemiconset and 70 hemorrhagic-onset patients with MMD, and found a significant association between hemorrhagic presentation with dilation and abnormal branching of the anterior choroidal artery. ${ }^{14}$ More recent high-resolution MRI studies suggested that these collateral vessels were the source of the hemorrhage. ${ }^{6,9}$ Based on these observations,

TABLE 3. Proportions (adjusted odds ratios) of grade 2 MMD in each collateral pattern

\begin{tabular}{|c|c|c|c|c|c|c|c|c|c|c|}
\hline \multirow[b]{2}{*}{ Collateral } & \multicolumn{2}{|c|}{ Grade $\leq 1$} & \multicolumn{2}{|c|}{ Grade 2} & \multicolumn{3}{|c|}{ Crude } & \multicolumn{3}{|c|}{ Sex/Age Adjusted } \\
\hline & No. & $\%$ & No. & $\%$ & OR & $95 \% \mathrm{Cl}$ & $p$ Value & OR & $95 \% \mathrm{Cl}$ & $p$ Value \\
\hline \multicolumn{11}{|c|}{ Lenticulostriate anastomosis } \\
\hline Ischemic & 80 & $77.7 \%$ & 23 & $22.3 \%$ & 1.00 & Reference & & 1.00 & Reference & \\
\hline Hemorrhagic & 54 & $72.0 \%$ & 21 & $28.0 \%$ & 1.35 & $0.68-2.68$ & 0.387 & 1.88 & $0.89-3.96$ & 0.097 \\
\hline \multicolumn{11}{|c|}{ Thalamic anastomosis } \\
\hline Ischemic & 86 & $83.5 \%$ & 17 & $16.5 \%$ & 1.00 & Reference & & 1.00 & Reference & \\
\hline Hemorrhagic & 53 & $70.7 \%$ & 22 & $29.3 \%$ & 2.10 & $1.02-4.31$ & 0.043 & 2.18 & $1.03-4.60$ & 0.041 \\
\hline \multicolumn{11}{|c|}{ Choroidal anastomosis } \\
\hline Ischemic & 86 & $83.5 \%$ & 17 & $16.5 \%$ & 1.00 & Reference & & 1.00 & Reference & \\
\hline Hemorrhagic & 40 & $53.3 \%$ & 35 & $46.7 \%$ & 4.43 & $2.22-8.83$ & $<0.001$ & 4.74 & $2.31-9.72$ & $<0.001$ \\
\hline
\end{tabular}

Overall 178 hemispheres ( $n=103$ ischemic and 75 hemorrhagic). 
TABLE 4. Proportions (adjusted odds ratios) of PCA involvement

\begin{tabular}{|c|c|c|c|c|c|c|c|c|c|c|}
\hline \multirow[b]{3}{*}{ MMD Group } & \multicolumn{4}{|c|}{ PCA Involvement } & & & & & & \\
\hline & \multicolumn{2}{|c|}{ Grade 0} & \multicolumn{2}{|c|}{ Grade $\geq 1$} & \multicolumn{3}{|c|}{ Crude } & \multicolumn{3}{|c|}{ Sex/Age Adjusted } \\
\hline & No. & $\%$ & No. & $\%$ & OR & $95 \% \mathrm{Cl}$ & $p$ Value & OR & $95 \% \mathrm{Cl}$ & $p$ Value \\
\hline Ischemic & 81 & $78.6 \%$ & 22 & $21.4 \%$ & 1.00 & Reference & & 1.00 & Reference & \\
\hline Hemorrhagic & 51 & $68.0 \%$ & 24 & $32.0 \%$ & 1.73 & $0.88-3.41$ & 0.111 & 1.60 & $0.79-3.21$ & 0.189 \\
\hline
\end{tabular}

Overall 178 hemispheres ( $n=103$ ischemic and 75 hemorrhagic).

we specifically defined choroidal collaterals (anastomosis) as the anastomosis between the choroidal and medullary arteries, and classified the extent of this collateral by intrinsic grading (Table 1). Our results further support previous observations that the development of choroidal anastomosis is a critical factor underlying hemorrhagic presentation in adult MMD. Regarding angiographic staging, the present study provided an interesting finding that $\mathrm{Su}-$ zuki's angiographic staging was significantly higher in the hemorrhagic-onset group than that in the ischemic-onset group, as indicated by a higher proportion of patients beyond Suzuki stage $4 .{ }^{16}$ Since abnormal vascular networks at the base of the brain start to decrease in size at stage 4 , and ultimately disappear at stages 5 and 6 , the higher angiographic stages in hemorrhagic-onset patients in the present study were apparently unique. ${ }^{15,16}$ We speculate that a lengthy period during which basal collaterals are maintained, including thalamic and/or choroidal anastomosis, might ultimately result in hemorrhagic presentation.

The JAM Trial and its supplemental analyses not only demonstrated the efficacy of direct EC-IC bypass for hemorrhagic-onset MMD, ${ }^{13}$ but also provided important information regarding the mechanisms underlying the hemorrhagic presentation in adult MMD. ${ }^{7,17}$ A prespecified subgroup analysis of the JAM Trial indicated, for the first time, that the annual rebleeding rate of patients with the posterior hemorrhage was as high as $17.1 \%$ and was significantly higher than that of patients with anterior circulation hemorrhage (3.0\%), and EC-IC bypass effectively reduced the risk of rebleeding exclusively in patients with posterior hemorrhage. ${ }^{17} \mathrm{We}$ further conducted a cross-sectional study using the data set of the JAM Trial and found that choroidal anastomosis and PCA involvement are characteristic of posterior hemorrhage in MMD. ${ }^{7}$ Therefore, we speculated that these factors, including the development of choroidal anastomosis, could also explain the high risk of hemorrhagic presentation in MMD. In fact, our results indicated the significance of choroidal and thalamic collateral development as a characteristic finding of hemorrhagic-onset MMD in comparison with ischemic-onset
MMD. Alternatively, we did not find any differences in PCA involvement between the onset groups, which could be explained by the observation that PCA stenosis/occlusion also contributed significantly to ischemic pathology in MMD. 8,12

Our results provide critical information for surgical strategies for adult MMD. EC-IC bypass, including superficial temporal artery-middle cerebral artery anastomosis with or without indirect pial synangiosis, is currently a standard management option for MMD patients with ischemic symptoms,$^{15}$ and its indication has been expanded to MMD patients with posterior hemorrhage. ${ }^{17}$ The JAM Trial and its supplemental analyses, including the present study, have suggested that patterns with choroidal and thalamic collaterals are different between each onset type, and the development of choroidal anastomosis is one of the most critical detrimental factors to induce bleeding/ rebleeding., ${ }^{717}$ These findings together suggest that temporal artery-middle cerebral artery anastomosis could reduce the risk of rebleeding in hemorrhagic-onset MMD by ameliorating the hemodynamic stress to the choroidal and thalamic collaterals. Although the exact efficacy of supplemental indirect pial synangiosis to direct EC-IC bypass for hemorrhagic MMD is undetermined ${ }^{11}$ increasing evidence suggests the superiority of combined direct/ indirect revascularization surgery in ischemic-onset adult MMD. ${ }^{1,2}$ Future comparative study between direct revascularization and direct/indirect combined revascularization for hemorrhagic-onset MMD patients will address this important issue.

The present study has the following limitations. First, the patients' age was significantly higher in the hemorrhagic-onset group than in the ischemic-onset group. Second, we recruited the angiographic data of the ischemiconset MMD patients separately from the JAM Trial, and the image-determination committee was not blinded to the patient onset type in the present study. Furthermore, the ischemic-onset MMD patients were recruited from 4 academic institutes among 22 centers that participated in the JAM Trial. Therefore, we cannot completely rule out

TABLE 5. Comparison of Suzuki angiographic stage between ischemic-onset and hemorrhagic-onset groups

\begin{tabular}{|c|c|c|c|c|c|c|c|c|c|c|}
\hline \multirow[b]{3}{*}{ MMD Group } & \multicolumn{4}{|c|}{ Suzuki Stage } & & & & & & \\
\hline & \multicolumn{2}{|c|}{ Grade $<4$} & \multicolumn{2}{|c|}{ Grade $\geq 4$} & \multicolumn{3}{|c|}{ Crude } & \multicolumn{3}{|c|}{ Sex/Age Adjusted } \\
\hline & No. & $\%$ & No. & $\%$ & OR & $95 \% \mathrm{Cl}$ & $p$ Value & OR & $95 \% \mathrm{Cl}$ & $p$ Value \\
\hline Ischemic & 49 & $47.6 \%$ & 54 & $52.4 \%$ & 1.00 & Reference & & 1.00 & Reference & \\
\hline Hemorrhagic & 24 & $32.0 \%$ & 51 & $68.0 \%$ & 1.93 & $1.04-3.59$ & 0.038 & 1.95 & $1.02-3.74$ & 0.045 \\
\hline
\end{tabular}

Overall 178 hemispheres ( $n=103$ ischemic and 75 hemorrhagic). 
the possibility of bias regarding patient selection and/or decision determining the angiographic grade. Finally, we were not able to evaluate the results of external carotid artery angiography because the JAM Trial did not include this information. In light of the significant contribution of transdural anastomosis from the external carotid artery system in MMD patients beyond Suzuki stage $4,{ }^{4,16}$ the contribution of the spontaneously developed pial synangiosis to cerebral hemodynamics could affect the development of each collateral in patients with higher Suzuki angiographic staging. As our result also indicated that the incidence of advanced Suzuki staging (stage 4-6) was significantly higher in the hemorrhagic-onset group, it would be of great interest to compare the development of transdural anastomosis in the external carotid artery system between each onset type.

\section{Conclusions}

In adult MMD, the characteristic pattern of abnormal vascular networks at the base of the brain is different between each onset type. The hemorrhagic-onset patients showed a significantly higher proportion of thalamic and choroidal anastomosis compared with ischemic-onset patients. Development of these collaterals, especially the choroidal collateral extending beyond the lateral ventricle, may play a critical role in hemorrhagic presentation in MMD.

\section{Acknowledgments}

The JAM Trial has been funded since 1999 by a grant from the Japanese Ministry of Health, Labour and Welfare as a major project of the Research Committee on Spontaneous Occlusion of the Circle of Willis (moyamoya disease). This case-control study was supported by AMED Grant Number J170001344.

\section{References}

1. Bang JS, Kwon OK, Kim JE, Kang HS, Park H, Cho SY, et al: Quantitative angiographic comparison with the OSIRIS program between the direct and indirect revascularization modalities in adult moyamoya disease. Neurosurgery 70:625-633, 2012

2. Cho WS, Kim JE, Kim CH, Ban SP, Kang HS, Son YJ, et al: Long-term outcomes after combined revascularization surgery in adult moyamoya disease. Stroke 45:3025-3031, 2014

3. Fujimura M, Tominaga T: Current status of revascularization surgery for moyamoya disease: special consideration for its 'internal carotid-external carotid (IC-EC) conversion' as the physiological reorganization system. Tohoku J Exp Med 236:45-53, 2015

4. Fujimura M, Tominaga T: Lessons learned from moyamoya disease: outcome of direct/indirect revascularization surgery for 150 affected hemispheres. Neurol Med Chir (Tokyo) 52:327-332, 2012

5. Fukui M: Guidelines for the diagnosis and treatment of spontaneous occlusion of the circle of Willis ('moyamoya' disease). Research Committee on Spontaneous Occlusion of the Circle of Willis (Moyamoya Disease) of the Ministry of Health and Welfare, Japan. Clin Neurol Neurosurg 99 (Suppl 2):S238-S240, 1997

6. Funaki T, Fushimi Y, Takahashi JC, Takagi Y, Araki Y, Yoshida K, et al: Visualization of periventricular collaterals in moyamoya disease with flow-sensitive black-blood magnetic resonance angiography: preliminary experience. Neurol Med Chir (Tokyo) 55:204-209, 2015

7. Funaki T, Takahashi JC, Houkin K, Kuroda S, Takeuchi S, Fujimura M, et al: Angiographic features of hemorrhagic moyamoya disease with high recurrence risk: a supplementary analysis of the Japan Adult Moyamoya Trial. J Neurosurg 128:777-784, 2018

8. Funaki T, Takahashi JC, Takagi Y, Yoshida K, Araki Y, Kikuchi T, et al: Impact of posterior cerebral artery involvement on long-term clinical and social outcome of pediatric moyamoya disease. J Neurosurg Pediatr 12:626-632, 2013

9. Funaki T, Takahashi JC, Yoshida K, Takagi Y, Fushimi Y, Kikuchi T, et al: Periventricular anastomosis in moyamoya disease: detecting fragile collateral vessels with MR angiography. J Neurosurg 124:1766-1772, 2016

10. Irikura K, Miyasaka Y, Kurata A, Tanaka R, Fujii K, Yada K, et al: A source of haemorrhage in adult patients with moyamoya disease: the significance of tributaries from the choroidal artery. Acta Neurochir (Wien) 138:1282-1286, 1996

11. Jiang H, Ni W, Xu B, Lei Y, Tian Y, Xu F, et al: Outcome in adult patients with hemorrhagic moyamoya disease after combined extracranial-intracranial bypass. J Neurosurg 121:1048-1055, 2014

12. Miyamoto S, Kikuchi H, Karasawa J, Nagata I, Ikota T, Takeuchi S: Study of the posterior circulation in moyamoya disease. Clinical and neuroradiological evaluation. J Neurosurg 61:1032-1037, 1984

13. Miyamoto S, Yoshimoto T, Hashimoto N, Okada Y, Tsuji I, Tominaga $\mathrm{T}$, et al: Effects of extracranial-intracranial bypass for patients with hemorrhagic moyamoya disease: results of the Japan Adult Moyamoya Trial. Stroke 45:1415-1421, 2014

14. Morioka M, Hamada J, Kawano T, Todaka T, Yano S, Kai Y, et al: Angiographic dilatation and branch extension of the anterior choroidal and posterior communicating arteries are predictors of hemorrhage in adult moyamoya patients. Stroke 34:90-95, 2003

15. Research Committee on the Pathology and Treatment of Spontaneous Occlusion of the Circle of Willis: Guidelines for diagnosis and treatment of moyamoya disease (spontaneous occlusion of the circle of Willis). Neurol Med Chir (Tokyo) 52:245-266, 2012

16. Suzuki J, Takaku A: Cerebrovascular "moyamoya" disease. Disease showing abnormal net-like vessels in base of brain. Arch Neurol 20:288-299, 1969

17. Takahashi JC, Funaki T, Houkin K, Inoue T, Ogasawara K, Nakagawara J, et al: Significance of the hemorrhagic site for recurrent bleeding: prespecified analysis in the Japan Adult Moyamoya Trial. Stroke 47:37-43, 2016

\section{Disclosures}

The authors report no conflict of interest concerning the materials or methods used in this study or the findings presented in this paper.

\section{Author Contributions}

Conception and design: Houkin, Miyamoto. Acquisition of data: Fujimura, Funaki, Takahashi, Kuroda. Analysis and interpretation of data: Fujimura, Funaki, Takahashi, Kuroda, Miyamoto. Drafting the article: Fujimura. Critically revising the article: Funaki, Houkin, Kuroda, Tomata, Tominaga, Miyamoto. Statistical analysis: Tomata. Study supervision: Houkin, Tominaga, Miyamoto.

\section{Correspondence}

Miki Fujimura: Tohoku University Graduate School of Medicine, Sendai, Japan. fujimur@nsg.med.tohoku.ac.jp. 